C2008 IEEE. Personal use of this material is permitted. However, permission to reprint/republish this material for advertising or promotional purposes or for creating new collective works for resale or redistribution to servers or lists, or to reuse any copyrighted component of this work in other works must be obtained from the IEEE. 


\title{
Performance of RLMS Algorithm in Adaptive Array Beam Forming
}

\author{
Jalal Abdulsayed Srar and Kah-Seng Chung \\ Department of Electrical and Computer Engineering, Curtin University of Technology, Perth, WA \\ jalal.srar@postgrad.curtin.edu.au, and k.chung@curtin.edu.au
}

\begin{abstract}
This paper examines the performance of an adaptive linear array employing the new RLMS algorithm, which consists of a recursive least square (RLS) section followed by a least mean square (LMS) section. The performance measures used are output and input signal-to-interference plus noise ratios (SINR), side lobe level $(S L L)$, and $\triangle S I N R_{o}$ as a function of the direction of arrival of the interfering signal. Computer simulation results show that the performance of RLMS is superior to either the RLS or LMS based on these measures, particularly when operating with low input SINR.
\end{abstract}

Keywords - RLS algorithm, LMS algorithm, RLMS algorithm, adaptive antenna array beam forming.

\section{INTRODUCTION}

The continued demand for wireless communication services is spearheading research in new techniques for enhancing spectral utilization. One such technique is the use of adaptive or smart antennas to produce a movable beam pattern that can be directed to the desired coverage areas. This characteristic minimizes the impact of unwanted noise and interference, thereby improving the quality of the desired signal.

An adaptive antenna consists of an array of antenna elements. The signals picked up by these individual elements are combined through the use of a signal processing unit to form a beam pattern that can be steered toward the desired coverage direction [1]. The performance of the signal processing unit is generally dictated by the beam forming algorithm used. The LMS or RLS are two commonly used algorithms for adaptive beam forming. The former has good tracking performance with low computational complexity, and is robust against numerical errors. On the other hand, the RLS algorithm can achieve a faster convergence which is independent of the eigen-value spread variations of the input signal correlation matrix [1]. These desirable features offered by both the LMS and RLS algorithms can be jointly realized through the use of a new algorithm, called RLMS [2]. The RLMS algorithm consists of two signal processing sections; an RLS section followed by an LMS section, as shown in Fig. 1. The convergence performance of RLMS is analyzed in [2].

In this paper, the effectiveness of the RLMS algorithm for beam forming in an adaptive linear array consisting of $N$ isotropic antenna elements is evaluated under different operating conditions, including the presence of a cochannel interfering signal, and additive white Gaussian noise (AWGN) of zero mean and variance $\sigma^{2}$. The performance measures adopted are the signal-to-interference plus noise ratio $($ SINR $)$, the side lobe level $(S L L)$, and the variation of the output SINR as a function of the angle of arrival $(A O A)$ of the interfering signal. For comparison, corresponding results obtained with the use of only the RLS or LMS algorithm are also presented.

The paper is organized as follows. In section II, the RLMS system model for the adaptive array is described. Section III reviews the convergence of the RLMS algorithm. A description of the computer simulation study is provided in Section IV, followed by the results presented in Section V. Section IV concludes the paper.

\section{RLMS SYSTEM OVERVIEW}

Fig. 1 shows the block diagram of an $N$-isotropic element adaptive linear array, which employs RLMS as its beam forming algorithm.

Let the desired signal $s_{d}(t)$ and a cochannel interference $s_{i}(t)$, both originated from a distance, are impinging on the array at an angle $\theta_{d}$ and $\theta_{i}$, respectively, as shown in Fig. 1. The resulting outputs of the individual antenna elements in the presence of AWGN, $\mathbf{n}(t)$ of variance $\sigma^{2}$ can be expressed as

$$
\begin{aligned}
\mathbf{x}(t) & =\left[x_{1}(t), x_{2}(t), \ldots, x_{N}(t)\right]^{T} \\
& =\mathbf{A}_{d} s_{d}(t)+\mathbf{A}_{i} s_{i}(t)+\mathbf{n}(t)
\end{aligned}
$$

where $\mathbf{A}_{d}$ and $\mathbf{A}_{i}$ are the array factors for the desired signal and the cochannel interference, respectively. By referencing with respect to the first element, $\mathbf{A}_{d}$ and $\mathbf{A}_{i}$ are given by

$$
\begin{aligned}
& \boldsymbol{A}_{d}=\left[1, e^{-j \psi_{d}}, e^{-2 j \psi_{d}}, \ldots ., e^{-(N-1) j \psi_{d}}\right]^{T} \\
& \boldsymbol{A}_{i}=\left[1, e^{-j \psi_{I}}, e^{-2 j \psi_{I}}, \ldots ., e^{-(N-1) j \psi_{I}}\right]^{T}
\end{aligned}
$$

with $\psi_{d}=2 \pi\left(\frac{d \sin \left(\theta_{d}\right)}{\lambda}\right)$ and $\psi_{i}=2 \pi\left(\frac{d \sin \left(\theta_{i}\right)}{\lambda}\right)$, where $d$ is the antenna element spacing, $\lambda$ is the carrier wavelength $[3]$, and $(\bullet)^{T}$ denotes transpose. 


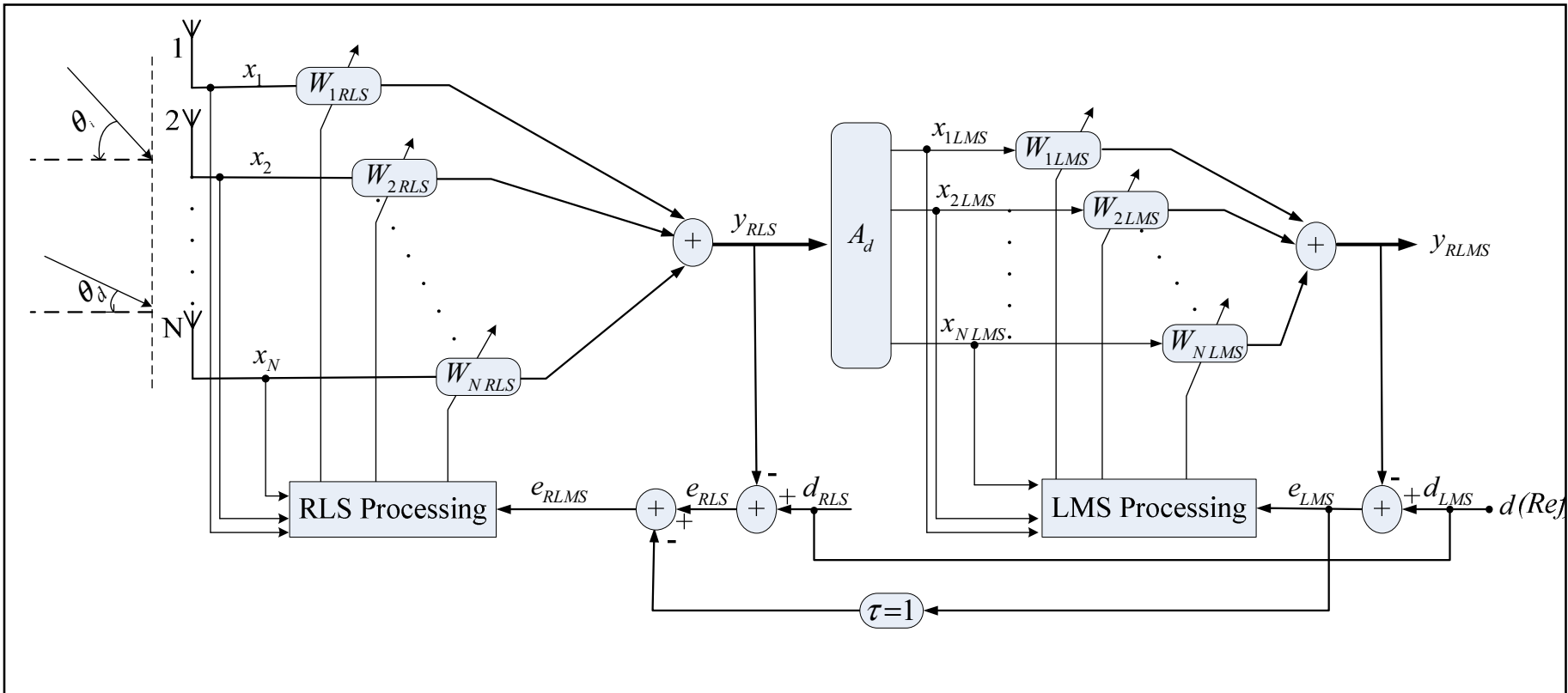

Figure 1. The block diagram of an adaptive array system employing the RLMS algorithm [2]

According to Fig. 1, the input stage of the RLMS scheme is based on the RLS algorithm with its weight vector at the $(j+1)^{t h}$ iteration updated according to [4]

$$
\boldsymbol{W}_{R L S}(j+1)=\boldsymbol{W}_{R L S}(j)+\boldsymbol{p}(j+1) \boldsymbol{X}(j) e_{R L S}^{*}(j) \boldsymbol{W}_{R L S}(j)
$$

where $\boldsymbol{p}(j)$ is an arbitrary symmetric positive definite matrix given by

$$
\boldsymbol{p}(j+1)=\frac{1}{\alpha}\left[\boldsymbol{p}(j)-\frac{\boldsymbol{p}(j) \boldsymbol{X}(j) \boldsymbol{X}^{H}(j) \boldsymbol{p}(j)}{\alpha+\boldsymbol{X}^{H}(j) \boldsymbol{p}(j) \boldsymbol{X}(j)}\right]
$$

$\boldsymbol{p}(j)$ is initialized by $\delta^{-1} \boldsymbol{I}$, with $\delta$ being a small positive constant, $\alpha$ is the forgetting factor and $\boldsymbol{I}$ is an $N \times N$ unity matrix.

Now, the output of the RLS section at the $j^{\text {th }}$ iteration can be expressed as

$$
y_{R L S}(j)=\boldsymbol{W}_{R L S}^{H}(j) \boldsymbol{X}(j)
$$

where $\boldsymbol{W}^{\boldsymbol{H}}(\cdot)$ denotes the complex conjugate transpose of the weight vector $\boldsymbol{W}(\cdot)$.

With this signal forming the input to the following LMS section, the input signal vector of the LMS section becomes

$$
\boldsymbol{X}_{L M S}=\boldsymbol{A}_{\boldsymbol{d}} y_{R L S}=\boldsymbol{A}_{\boldsymbol{d}} \boldsymbol{W}_{R L S}^{H} \boldsymbol{X}
$$

For the LMS stage, its weight vector is updated according to

$$
\boldsymbol{W}_{L M S}(j+1)=\boldsymbol{W}_{L M S}(j)+\mu \boldsymbol{X}_{L M S}(j) e_{L M S}(j) \quad 0<\mu<\mu_{0}
$$

where $\mu_{0}$ is a positive number that depends on the input signal statistics.

Finally, the output of the RLMS beam former is given by

$$
\begin{aligned}
y_{R L M S} & =\boldsymbol{W}_{L M S}^{H} \boldsymbol{X}_{\boldsymbol{L M S}} \\
& =\boldsymbol{W}_{L M S}^{H} \boldsymbol{A}_{\boldsymbol{d}} \boldsymbol{W}_{R L S}^{H} \boldsymbol{X} \\
& \equiv \boldsymbol{W}_{R L M S}^{H} \boldsymbol{X}
\end{aligned}
$$

\section{CONVERGENCE OF THE RLMS ALGORITHM}

The convergence of the RLMS algorithm can be studied by observing its mean-square error $\xi$, defined as

$$
\xi \triangleq E\left[\left|e_{R L M S}\right|^{2}\right]
$$

where $E[]$ denotes the expectation operator and $|\bullet|$ signifies the modulus.

From Fig. 1, the overall error signal for the RLMS algorithm at the $j^{\text {th }}$ iteration is given by

$$
e_{R L M S}(j)=e_{R L S}(j)-e_{L M S}(j-1)
$$


with

$$
\begin{aligned}
& e_{R L S}(j)=d_{R L S}(j)-\boldsymbol{W}_{R L S}^{H}(j) \boldsymbol{X}(j) \\
& e_{L M S}(j)=d_{L M S}(j)-\boldsymbol{W}_{L M S}^{H}(j) \boldsymbol{X}_{L M S}(j)
\end{aligned}
$$

where $d_{R L S}$ and $d_{L M S}$ correspond to the reference signals for the RLS and LMS sections, respectively.

Applying (11) and (12) to (10), we obtain

$$
\begin{aligned}
\xi= & E\left\{\left[e_{R L S}(i)-e_{L M S}(i-1)\right]^{2}\right\} \\
= & \sum_{i=1}^{j} \lambda^{j-i} E\left\{\left[d_{R L S}(i)-\boldsymbol{W}_{R L S}^{H}(j) \boldsymbol{X}(i)-e_{L M S}(i-1)\right]^{2}\right\} \\
= & \sum_{i=1}^{j} \lambda^{j-i}\left\{E\left[D^{2}(i)\right]-2 E\left[D(i) \boldsymbol{X}^{H}(i) \boldsymbol{W}_{R L S}(j)\right]\right\}+ \\
& \boldsymbol{W}_{R L S}^{H}(j) \boldsymbol{Q}(j) \boldsymbol{W}_{R L S}(j)
\end{aligned}
$$

where $D(j)=d_{R L S}(j)-e_{L M S}(j-1)$, and $\boldsymbol{Q}$ is the correlation matrix of the input signals given by [5] as

$$
\boldsymbol{Q}(j)=\sum_{i=1}^{j} \lambda^{j-i} \boldsymbol{X}(j) \boldsymbol{X}^{H}(j)
$$

Since $\boldsymbol{W}_{R L M S}^{H}=\boldsymbol{W}_{L M S}^{H} \boldsymbol{A}_{\boldsymbol{d}} \boldsymbol{W}_{R L S}^{H}$, it has been shown in [2] that the summation terms on the RHS of (13) are given by

$$
\begin{aligned}
& \sum_{i=1}^{j} \lambda^{j-i}\left\{E\left[D^{2}(i)\right]\right\}=\sum_{i=1}^{j} \lambda^{j-i}\left\{\left|d_{R L S}(i)\right|^{2}+\left|d_{L M S}(i-1)\right|^{2}\right\} \\
& -2 \boldsymbol{W}_{R L M S}^{H}(j-1) \boldsymbol{Z}(j-1)+\boldsymbol{W}_{R L M S}^{H}(j-1) \boldsymbol{Q}(j-1) \boldsymbol{W}_{R L M S}(j-1)
\end{aligned}
$$

and

$$
\sum_{i=1}^{j} \lambda^{j-i}\left\{-2 E\left[D(i) \boldsymbol{X}^{H}(i) \boldsymbol{W}_{R L S}(j)\right]\right\}=-2 \boldsymbol{Z}^{H}(j) \boldsymbol{W}_{R L S}(j)
$$

where $\boldsymbol{Z}(j)$ is the input signal cross-correlation vector given by [5] as

$$
\boldsymbol{Z}(j)=\sum_{i=1}^{j} \lambda^{j-i} \boldsymbol{X}(j) d^{*}(j)
$$

As a result, the mean square error $\xi$ as specified by (13) can be rewritten as

$$
\begin{aligned}
\boldsymbol{\xi}=\sum_{i=1}^{j} \lambda^{j-i}\left\{\left|d_{R L S}(i)\right|^{2}+\left|d_{L M S}(i-1)\right|^{2}\right\}-2 \boldsymbol{W}_{R L M S}^{H}(j-1) \boldsymbol{Z}(j-1)+ \\
\\
\boldsymbol{W}_{R L M S}^{H}(j-1) \boldsymbol{Q}(j-1) \boldsymbol{W}_{R L M S}(j-1)-2 \boldsymbol{Z}^{H}(j) \boldsymbol{W}_{R L S}(j)+ \\
\boldsymbol{W}_{R L S}^{H}(j) \boldsymbol{Q}(j) \boldsymbol{W}_{R L S}(j)
\end{aligned}
$$

The optimal weight vector $\boldsymbol{W}_{\text {opt } t_{R S}}(j)$ is obtained by first differentiating (18) with respect to $\boldsymbol{W}_{R L S}^{H}(j)$ yielding the gradient vector $\nabla(\xi)$. After equating $\nabla(\xi)$ to zero, we obtain

$$
\boldsymbol{W}_{o p t_{R L S}}(j)=\boldsymbol{Q}^{-1}(j) \boldsymbol{Z}(j)
$$

With this optimal weight vector, the minimum value of the mean square error becomes

$$
\begin{aligned}
\xi_{\min } & =\sum_{i=1}^{j} \lambda^{j-i}\left\{\left|d_{R L S}(i)\right|^{2}+\left|d_{L M S}(i-1)\right|^{2}\right\}-\boldsymbol{Z}^{H}(j) \boldsymbol{W}_{\text {opt }_{R L S}}(j) \\
& +\boldsymbol{W}_{R L M S}^{H}(j-1) \boldsymbol{Z}(j-1)\left\{-2+\boldsymbol{A}_{d}^{H} \boldsymbol{W}_{L M S}(j-1)\right\}
\end{aligned}
$$

Furthermore, it is shown in [2] that as the adaptation progresses, the mean square error will eventually converge to

$$
\lim _{j \rightarrow \infty} \xi(j)=\xi_{\min }
$$

\section{Performance Study}

The performance of the RLMS algorithm has been studied by means of MATLAB simulation for an adaptive linear array consisting of eight isotropic antenna elements, spaced half carrier wavelength apart. For the simulations, the desired BPSK signal arrives at an angle $\theta_{d}=0^{\circ}$. It is corrupted by an interfering BPSK signal arriving at $\theta_{i}=45^{\circ}$ in the presence of AWGN of zero mean and variance $\sigma^{2}$. All the tap weights are initially set to zero. The forgetting factor used is $\alpha=1$, and the step size for the LMS tap weights is $\mu=0.075$. Each simulation run involves 1000 iterations.

At each iteration, the output signal-to-interference plus noise ratio, $\operatorname{SINR}_{o}(j)$ is calculated according to

$$
\operatorname{SINR}_{o}(j)=\frac{P_{d}(j)}{P_{i}(j)+P_{n}(j)}
$$

$$
\begin{array}{ll} 
& P_{d}(j)=\frac{V_{S}^{2}}{2}\left|\mathbf{W}^{H}(j) \mathbf{A}_{d}\right|^{2} \\
\text { with } \quad P_{i}(j) & =\frac{V_{i}^{2}}{2}\left|\mathbf{W}^{H}(j) \mathbf{A}_{i}\right|^{2} \\
& P_{n}(j)=\sigma^{2}\left|\mathbf{W}^{H}(j)\right|^{2}
\end{array}
$$


where $P_{d}(j), P_{i}(j)$ and $P_{n}(j)$ are the average output powers, at $j^{\text {th }}$ iteration, of the desired signal, the interference signal and the AWGN, respectively. $V_{s}$ and $V_{i}$ are the input amplitudes of the desired and interfering BPSK signals, respectively. For the simulation, $V_{s}$ is equal to 1 Volt, and $\sigma$ is the rms noise voltage. $\mathbf{W}^{H}(j)$ is as defined in (6).

For comparison purposes, simulations have also been repeated using either the LMS or RLS algorithm on its own.

\section{Simulation Results}

The performance of the RLMS scheme is evaluated according to the following measures:

- Signal-to-interference plus noise ratio (SINR)

- $\quad$ Side lobe level $(S L L)$

- $\triangle S I N R_{o}$ against angle of arrival of the interference.

\section{A. Output SINR versus input SINR :}

The influence of interference and noise on the performance of the RLMS algorithm has been evaluated in terms of the $S I N R_{o}$ achieved after convergence as a function of the input SINR. Fig. 2 shows the resultant $\operatorname{SINR}_{o}$ achieved with the RLMS, LMS and RLS algorithms over an input SINR range of -5 to $10 \mathrm{~dB}$ with $\sigma=0.01$. The effect of a larger $\sigma$ of 0.05 is shown in Fig. 3. From Fig. 2 and Fig. 3, it is obvious that the RLMS schemes out performs both the RLS and LMS algorithms in terms of achievable $\operatorname{SINR}_{o}$. Also, it is observed that the RLMS scheme achieves a larger $\operatorname{SINR}_{o}$ for a given input SINR when $\sigma$ is larger. This suggests that the RLMS algorithm is more sensitive to a change in interference level than noise. On the other hand, both the RLS and LMS algorithms tend to suffer from an increase in the noise level.

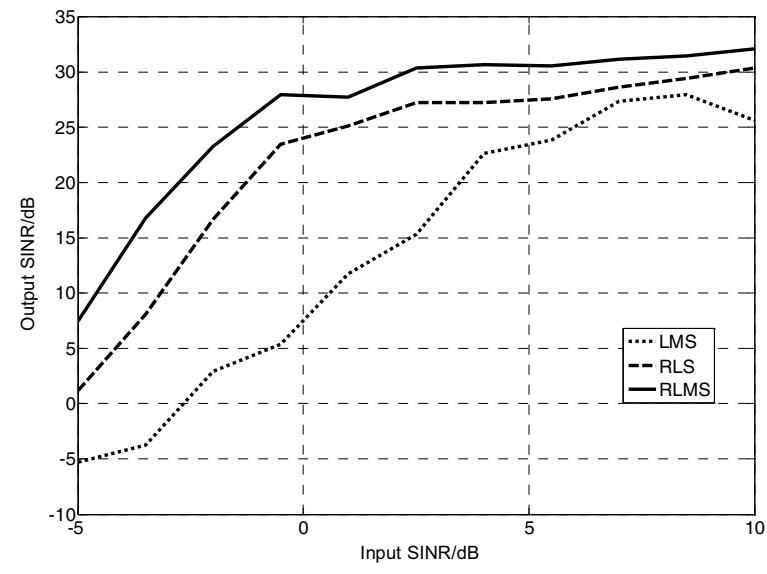

Figure 2. Output SINR versus input SINR with $\sigma=0.01$

\section{B. Beam pattern characteristics}

Fig. 4 shows the beam patterns obtained through the use of RLMS, RLS and LMS algorithms when the input SINR is 10 $\mathrm{dB}$ and $\sigma=0.05$. The maximum gain corresponds to the direction of arrival of the desired signal, i.e., $\theta_{d}=0$. Here, the side lobe level $(S L L)$ is defined as

$$
S L L(d B)=\text { Maximum side lobe gain }- \text { Gain at } \theta_{d}
$$

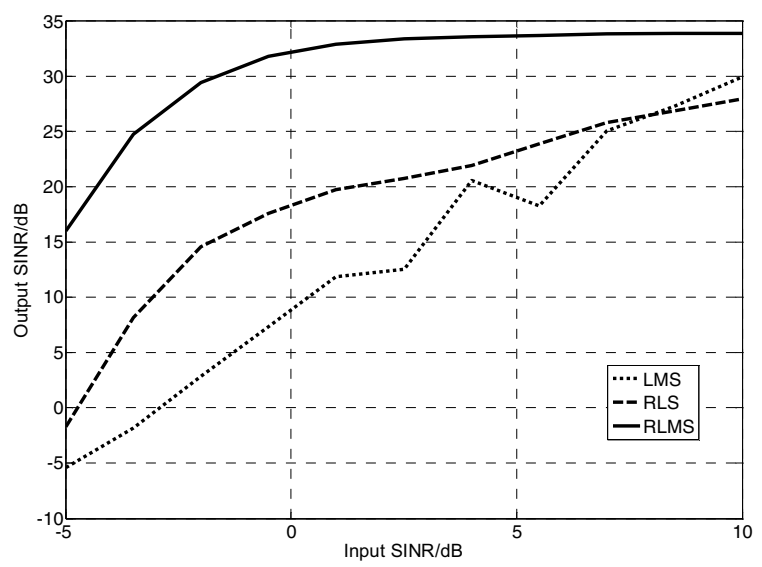

Figure 3. Output SINR versus input SINR with $\sigma=0.05$

For each of the three algorithms considered, $S L L$ values are obtained for a range of input SINR, extending from 0 to 15 $\mathrm{dB}$, with $\sigma=0.05$. These $S L L$ values are tabulated in Table 1 . It is observed that the three algorithms achieve similar $S L L$ performance when input SINR is larger than $10 \mathrm{~dB}$. However, the RLMS scheme is far superior at lower input SINR. Based on this SLL measure, it is clear that the RLMS scheme achieves the best performance among the three algorithms considered.

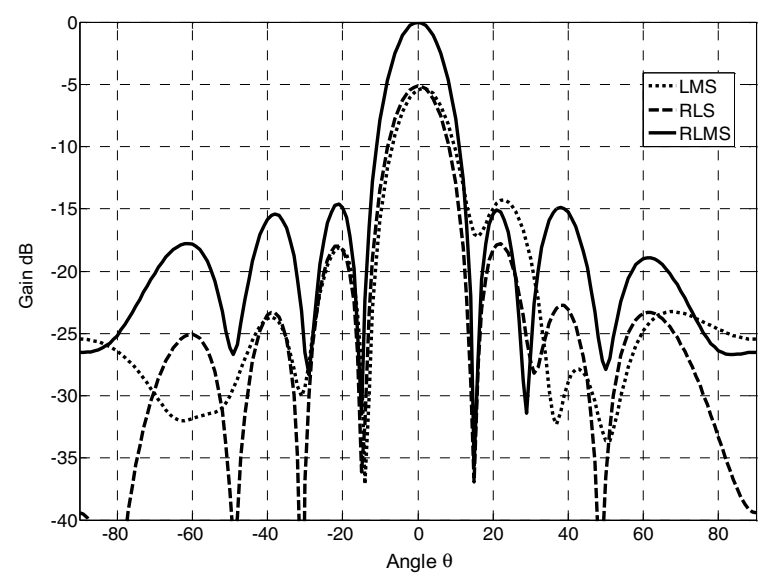

Figure 4. The beams patterns obtained with LMS, RLS and RLMS algorithms (input $\operatorname{SINR}=10 \mathrm{~dB}$ with $\sigma=0.05$ ) 
TABLE I. SLL (DB) ACHIEVED AT DIFFERENT INPUT SINRS (DB)

\begin{tabular}{|c|c|c|c|c|c|c|c|}
\hline \multirow{2}{*}{ Algorithm } & \multicolumn{7}{|c|}{ Input SINR (dB) } \\
& 0 & 2.5 & 5 & 7.5 & 10 & 12.5 & 15 \\
\hline RLMS & -16.27 & -14.36 & -14.38 & -14.9 & -13.65 & -12.32 & -12.21 \\
\hline RLS & -13.99 & -12.87 & -12.37 & -12.2 & -11.85 & -11.22 & -12.11 \\
\hline LMS & -6 & -8 & -9.7 & -9.8 & -11 & -9.2 & -11.5 \\
\hline
\end{tabular}

\section{C. $\triangle$ SINR against AOA of the interference, $\theta_{i}$}

The influence of the direction of arrival of the interfering signal on the output SINR is also investigated. For this study, the desired signal has an input SINR of either $0 \mathrm{~dB}$ or $10 \mathrm{~dB}$ with $\sigma=0.05$. The interfering signal arrives at an angle $\theta_{i}$, which varies from $-90^{\circ}$ to $90^{\circ}$.

In this study, the performance measure adopted is

$$
\Delta \operatorname{SINR}_{o}=\operatorname{SINR}_{o, R L M S}-\operatorname{SINR}_{o, R L S}
$$

where $\operatorname{SINR}_{o, R L M S}$ and $\operatorname{SINR_{o,RLS}}$ are the ensemble average output SINR, obtained from 30 simulation runs, for the RLMS and RLS algorithms, respectively.

Fig. 5 shows the variation of $\triangle \operatorname{SINR}_{o}$ with $\theta_{i}$ for the case that the desired signal arrives at $\theta_{d}=0^{\circ}$, i.e., bore-side. The same results but obtained with $\theta_{d}=90^{\circ}$, i.e., end-fire, are plotted in Fig. 6. For both the bore-side and end-fire cases, it is noted that the RLMS scheme performs better than the RLS algorithm, i.e., the $\triangle S I N R_{o}$ values achieved are positive, except for a small region when $\left|\theta_{i}\right|>75^{\circ}$. It is possible for the RLMS scheme to achieve a larger gain in $\operatorname{SINR} R_{o}$ over the RLS algorithm when the input SINR drops from $10 \mathrm{~dB}$ to $0 \mathrm{~dB}$.

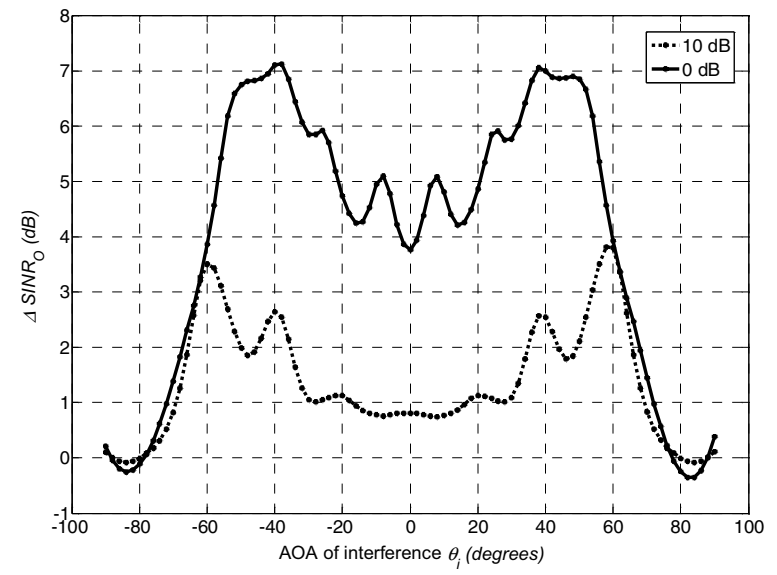

Figure 5. Changes of $\triangle S I N R_{o}$ with the $A O A$ of the interference. The desired signal arrives at $\theta_{d}=0^{\circ}$ and its input SINR is (i) $0 \mathrm{~dB}$ and (ii) $10 \mathrm{~dB}$ with $\sigma=0.05$.

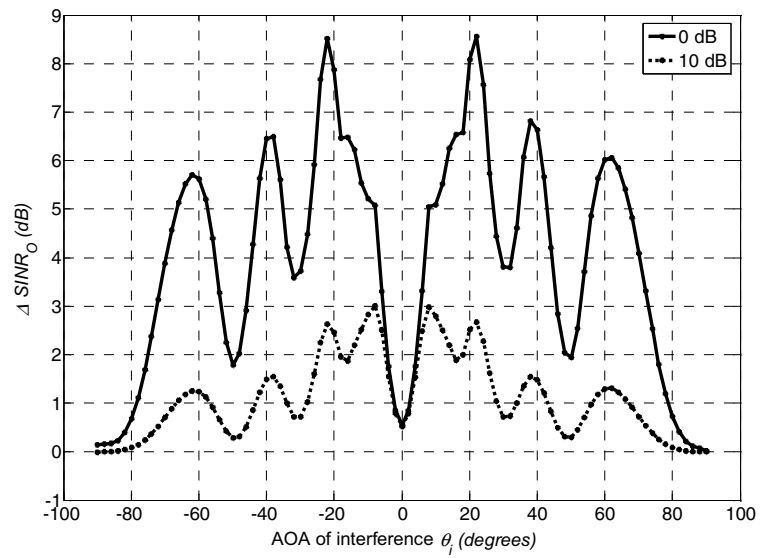

Figure 6. Changes of $\triangle S I N R_{o}$ with the AOA of the interference. The desired signal arrives at $\theta_{d}=90^{\circ}$ and its input SINR is (i) $0 \mathrm{~dB}$ and (ii) $10 \mathrm{~dB}$ with

$$
\sigma=0.05 \text {. }
$$

Also, the $\triangle \operatorname{SINR} R_{o}$ tends to peak at around $\theta_{i}= \pm 50^{\circ}$ for the bore-side case, and at around $\theta_{i}= \pm 20^{\circ}$ for the end-fire case.

\section{Performance with a noisy reference signal}

The performances of the RLMS, RLS and LMS schemes have also been studied when the reference signal used is corrupted by AWGN. This involves examining the effect on the mean square error $\xi$ as a result of varying the noise component in the reference signal. Fig. 7 shows the ensemble average of the mean square error, $\bar{\xi}$, obtained from 100 individual simulation runs, as a function of the ratio of the rms noise to the reference signal level.

From Fig. 7, it is observed that the RLMS scheme is the least sensitive to a noisy reference signal among the three algorithms considered. This is particularly true when the noise level is larger than 0.3 times the reference signal.

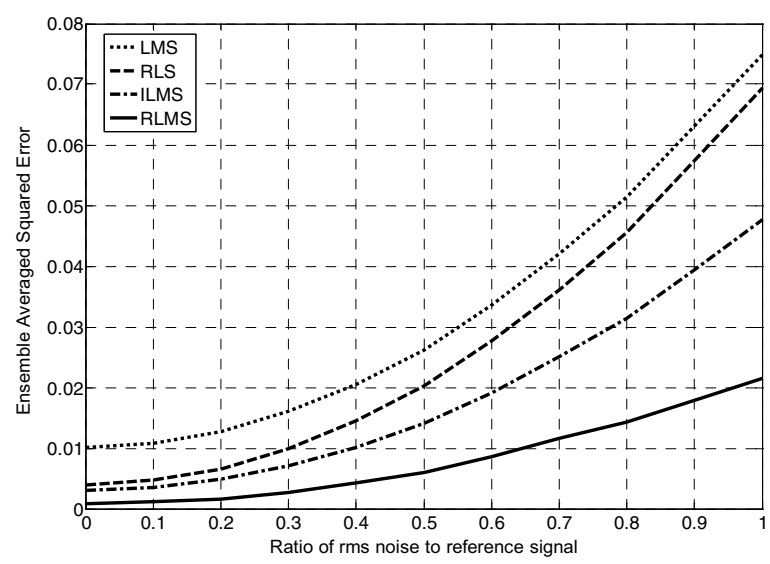

Figure 7. The influence of noise in the reference signal on the mean square error $\xi$. 


\section{CONCLUSIONS}

This paper compares the performance of digital beam forming using the RLMS, RLS and LMS algorithms. It is shown that the RLMS scheme outperforms the other two algorithms in all the performance measures considered in this paper, i.e., achievable output SINR, side lobe level, and influence of the AOA of the interference on the $S I N R_{o}$. In most cases, the RLMS scheme achieves a larger enhancement in performance at lower input SINR. Furthermore, it is shown that the RLMS algorithm is also more robust when the reference signal used is noisy. The RLMS algorithm complexity is slightly higher than that of the RLS algorithm as the complexity for the LMS is very low.

\section{REFERENCES}

[1] F.-B. Ueng, J.-D. Chen, and S.-H. Cheng, "Smart Antenna for Multiuser DS/CDMA Communication in Multipath Fading Channels," IEICE Transaction Communication, vol. E88, pp. 2944-2954, Jul 2005.

[2] J. A. Srar and K.-S. Chung, "Adaptive Array Beam Forming Using a Combined RLS-LMS Algorithm," in APCC2008 Japan, 2008.

[3] V. V. Zaharov, F. S. Casco, and O. A. Amin, "The tracking algorithm and processor for smart antenna cellular communications," in Microwave Electronics: Measurements, Identification, Application Conference, 2001. MEMIA 2001, 2001, pp. 175-181.

[4] L. C. Godara, "Application of antenna arrays to mobile communications. II. Beam-forming and direction-of-arrival considerations," Proceedings of the IEEE, vol. 85, pp. 1195-1245, 1997.

[5] S. Haykin, Adaptive Filter Theory, Third ed.: Prentic Hall, 1996. 